\title{
BMJ Open Systematic and continuous collection of patient-reported outcomes and experience in women with cancer undergoing mastectomy and immediate breast reconstruction: a study protocol for the Tuscany Region (Italy)
}

To cite: Ferrè F, De Rosis S, Murante AM, et al. Systematic and continuous collection of patient-reported outcomes and experience in women with cancer undergoing mastectomy and immediate breast reconstruction: a study protocol for the Tuscany Region (Italy). BMJ Open 2021;11:e042235. doi:10.1136/ bmjopen-2020-042235

- Prepublication history and additional materials for this paper are available online. To view these files, please visit the journal online (http://dx.doi. org/10.1136/bmjopen-2020042235).

Received 01 July 2020 Revised 29 November 2020 Accepted 10 December 2020

Check for updates

(c) Author(s) (or their employer(s)) 2021. Re-use permitted under CC BY-NC. No commercial re-use. See rights and permissions. Published by BMJ.

For numbered affiliations see end of article.

Correspondence to Dr Francesca Ferrè; francesca.ferre@santannapisa.it

\begin{abstract}
Francesca Ferrè (D) ," Sabina De Rosis (D) , Anna Maria Murante, ${ }^{1}$ Kendall Jamieson Gilmore, ${ }^{1}$ Matteo Ghilli, ${ }^{2}$ Donatella Mariniello, ${ }^{2}$ Sabina Nuti, Manuela Roncella ${ }^{2}$
\end{abstract}

\begin{abstract}
Introduction Monitoring how patients feel and what they experience during the care process gives health professionals data to improve the quality of care, and gives health systems information to better design and implement care pathways. To gain new insights about specific gaps and/or strengths in breast cancer care, we measure patient-reported outcomes (PROs) and patient-reported experiences (PREs) for women receiving immediate breast reconstruction (iBR).

Methods and analysis Prospective, multicentre, cohort study with continuous and systematic web-based data collection from women diagnosed with breast cancer, who have an indication for iBR after mastectomy treated at any Breast Unit (BU) in Tuscany Region (Italy). Patients are classified into one of two groups under conditions of routine clinical practice, based on the type of iBR planned

Strengths and limitations of this study

Aggregated patient-reported data to support healthcare system improvements and inform discussions with patients about treatment options-but which cannot be used prognostically with individual patients.

- Continuous and systematic web-based data collection.

- Multicentre study that allows benchmarking among providers.

- The regional-based sample limits the generalisability of findings.

- Health professionals when recruiting patients-if not properly engaged and motivated-could limit enrolment in the study.
\end{abstract} (implant and autologous reconstruction). Patient-reported information are obtained prior to and after surgery (at 3-month and 12-month follow-up). We estimate that there are around 700 annual eligible patients.

Descriptive analyses are used to assess trends in PROs over time and differences between types of iBR in PROs and PREs. Additionally, econometric models are used to analyse patient and BU characteristics associated with outcomes and experiences. PREs are evaluated to assess aspects of integrated care along the care pathway. Ethics and dissemination The study has been reviewed and obtained a nihil obstat from the Tuscan Ethics Committees of the three Area Vasta in 2017. Dissemination of results will be via periodic report, journal articles and conference presentations.

\section{INTRODUCTION}

Breast cancer is the most frequent malignant tumour among women. In Italy, the incidence is 53000 new cases per year, representing the most diagnosed malignancy in women, with an estimated prevalence of 800000 cases.
The long-term prognosis for women with breast cancer has improved significantly in recent decades, particularly due to enhanced early diagnosis and therapeutic innovations. The Italian 5-year survival rate fluctuates around $87 \%$, and the 10 -year survival around $80 \% .^{1}$ In line with this, surgical treatments for breast cancer have evolved over the past decades in an attempt to improve cosmetic outcomes and reduce surgical morbidity while ensuring an oncologically sound procedure. ${ }^{2}$ Clinical guidelines now recommend conservative surgical approaches, using oncoplastic techniques to ensure good cosmetic outcomes. Where mastectomy is required, conservative mastectomies and reconstruction should be available and offered to all women, with immediate breast reconstruction (iBR) preferred when possible. ${ }^{3}$ 
Ultimately, there is consensus that the optimal surgical approach for each patient should be discussed individually, considering anatomical treatment and patient-related factors and preferences. ${ }^{4}$ Breast cancer has a significant impact on patients' lives throughout the care pathway, rehabilitation and emotional-functional recovery. Optimising breast cancer outcomes remains a major challenge; what matters to patients should be considered as an important outcome.

Outcome indicators are increasingly used in measuring and monitoring quality, alongside process and structure indicators. This is particularly true for patient-reported outcomes (PROs) and patient-reported experiences (PREs), measured with patient-reported outcome measures (PROMs) and patient-reported experience measures (PREMs). ${ }^{5}$ PROMs typically address selfreported physical health, satisfaction and well-being, and social and emotional health, and can be administered at different points in time. A number of PRO surveys are in standard use, applying to specific conditions such as breast cancer or to general health and well-being.

PREMs are an additional source of information, complementary to PROMs, providing the patient's view of the delivery of services. These measures provide information about patients' feelings and experiences during the healthcare process. They are not a satisfaction score, instead seeking to elicit what actually occurred to patients receiving care, ${ }^{7-9}$ so providing managers and professionals with specific information on what 'does and does not work' from the perspective of patients. There is evidence that patient experience is associated with a range of relevant factors, including clinical safety, outcomes and cost. $^{1011}$

This information can be used by stakeholders for different purposes:

1. Patients, for whom PROs and PREs can be used in monitoring their health during treatment and followup, supporting improvements, and for whom aggregate data can indicate expected quality of life (QoL) from different treatment options. ${ }^{12} 13$

2. Health professionals, for whom feedback on their own delivered care, may facilitate personalisation and process improvements. ${ }^{1415}$

3. Healthcare providers who can use such information on a larger scale as a foundation for quality improvement. $^{16}$

4. Healthcare systems that can use such data to compare the outcomes of different providers and as part of studies to estimate health outcomes and cost-effectiveness of an intervention/treatment. ${ }^{17}$

In breast cancer care, PROs are increasingly collected as part of the treatment and recovery pathway, and have been associated with a range of positive effects including improved outcomes, improved experience, improved physician-patient interactions and higher patient activation. ${ }^{18} 19$

While the use of patient-reported data in breast cancer care is increasing, there remains a lack of standardisation in measures and collection methods. A recent systematic review of PROM administration methods in breast cancer found positive impacts from the systematic collection of PROMs, as well as highlighting a need for additional reporting of PROM collection frequency and methods. ${ }^{18}$ More broadly, published studies regarding satisfaction with breast cancer surgery are limited by small sample size, limited representativeness of institutions and surgeons, and the use of generic surveys not specifically designed for this population. ${ }^{20}$ Currently, the UK collects breast cancer PROs on a large scale as a national audit tool ${ }^{21}$; more recently the Organisation for Economic Co-operation and Development (OCED) has started international comparative work on breast cancer PROMs, involving international sites in data collection. ${ }^{22}$

PREs are also measured in breast cancer care, typically capturing patient experiences of specific aspects of care such as chemotherapy ${ }^{23}$ or follow-up programmes, ${ }^{24-26}$ or specific dimensions of patient experience such as patientdoctor communication. ${ }^{27}$ However, there are no available data on experience of patients with breast cancer from surgery through to follow-up.

Despite the importance of patient experience and outcomes in breast cancer reconstruction, few studies have been conducted where both PROs and PREs are collected. In wider oncology, the Dutch Head and Neck Cancer Audit provides an example of what is possible and desirable, ${ }^{28}$ while South East Scotland is implementing a regional platform for PROMs and PREMs in cancer, ${ }^{29}$ and other initiatives have started in National Health Service (NHS) Wales. ${ }^{30} 31$

This protocol describes the systematic collection of PROMs and PREMs from patients with breast cancer with indication for mastectomy and iBR in Tuscany Region (Italy). This is a multicentre study, with continuously enrolled patients providing self-reported outcomes and experience at multiple points in the care pathway, managed using a web platform which also enables realtime reporting of PRO and PRE data.

\section{OBJECTIVES}

The primary aims of this study are:

1. To describe PROs of overall satisfaction, psychosocial impact and physical well-being in women who undergo a mastectomy with planned iBR (implant and autologous reconstruction).

2. To analyse satisfaction and experience of care along the pathway using PRE data. Specifically, pathway assessments will focus on aspects of integrated care including organisation and accessibility, information and communication, and coordination and continuity.

3. To monitor, on behalf of the regional health system, provider participation in the patient-reported data collection programme. 
Table 1 Breast Units (BUs) included in the study

\begin{tabular}{|c|c|c|c|c|}
\hline Hospital type & Hospital full name & $\begin{array}{l}\text { No of breast cancer } \\
\text { reconstruction }(2019)^{*}\end{array}$ & $\begin{array}{l}\text { No of breast cancer } \\
\text { surgeries }(2019)^{\star}\end{array}$ & $\begin{array}{l}\text { No of professionals } \\
\text { working in the BU } \\
\text { (latest available data)† }\end{array}$ \\
\hline \multirow[t]{2}{*}{ Teaching hospital } & Azienda Ospedaliera Universitaria Pisana & 201 & 728 & 34 \\
\hline & Azienda Ospedaliera Universitaria Careggi & 160 & 876 & 40 \\
\hline \multirow{7}{*}{$\begin{array}{l}\text { Public hospital } \\
\text { managed by } \\
\text { the local health } \\
\text { authority }\end{array}$} & Nuovo Ospedale delle Apuane-Massa & 20 & 165 & NA \\
\hline & Ospedale Felice Lotti-Pontedera & 30 & 146 & NA \\
\hline & Ospedali Riuniti-Livorno & 49 & 220 & 14 \\
\hline & Ospedale Unico Versilia - Viareggio & 35 & 238 & 22 \\
\hline & Ospedale S Jacopo-Pistoia & 18 & 213 & 19 \\
\hline & Ospedale S M Annunziata-Firenze & 27 & 322 & 38 \\
\hline & Ospedale Misericordia-Grosseto & 13 & 180 & 15 \\
\hline Tuscany Region & & 703 & 4181 & 279 \\
\hline
\end{tabular}

*Performance evaluation system of Tuscany Region. ${ }^{54}$

tSenonetwork. $^{55}$

NA, not available.

\section{METHODS}

\section{Study design}

Prospective, multicentre, cohort study with continuous and systematic web-based recruitment of patients from January 2019. Data are digitally collected, stored and managed by the Management and Healthcare Laboratory, Scuola Superiore Sant'Anna, Pisa (Italy). Realtime reporting of aggregate data at site level is available through a web platform. PRO and PRE data are not currently linked to the Electronic Medical Records of patients or the Cancer Registry.

\section{Setting}

The study is performed in the 14 Breast Units (BUs) of Tuscany Region (Italy) listed in table 1. The Italian NHS is a regionally based system that provides universal coverage largely free of charge at the point of delivery. The regional healthcare system comprises three local health authorities (LHAs) with 38 district general hospitals directly managed by the LHAs, and four teaching hospitals (one paediatric). Since 2014, BUs are the only authorised providers for breast cancer surgery.

In the Italian NHS, breast cancer care is organised within recognised breast centres for which minimum standards are set. In seeking to reduce unwarranted variation and improve access to and utilisation of hospital services, services are centralised in higher volume centres with a minimum caseload of 150 newly diagnosed cases of primary breast cancer treated each year by each provider, in line with international scientific recommendations. ${ }^{2832}$

In Tuscany, around 4200 breast cancer surgeries are performed every year, of which about 700 are iBR after mastectomy (table 1). iBRs accounted for $60 \%$ of total mastectomies for breast cancer in 2019, with significant variation across hospitals (range 28\%-78\%) (figure 1).

Before study commencement, preliminary design and implementation activities were carried out with professionals and patients (figure 2). Phase 1, from 2016 to 2018 in the BU of the Teaching Hospital of Pisa, collected preoperative data in hospital using a tablet, with follow-up questionnaires issued by email. Results from the preliminary study justified the scale-up to regional level. At this point it was decided to additionally integrate PREMs in the data collection.

Phase 2-early engagement with other Tuscan BUscommenced in 2017, focused on identifying and sharing opportunities arising from the model and on practicalities of implementation. Based on lessons from phase 1 and elsewhere, ${ }^{33} 34$ clinicians were engaged throughout study design and roll-out. Site visits were held at each BU to map patient management processes in detail, identifying common time points for recruitment and baseline assessment across sites. During this phase the data platform and web-based recruitment tool were developed and presented to BUs. After a 2-week field trial, around $50 \%$ of Tuscan BUs participated in phase 3, a 1-year pilot study during 2018, focused on engagement with professionals and increasing BU participation. Following this pilot, the hosting web platform was updated alongside other minor adjustments in advance of phase 4 wider roll-out.

In 2019, the survey was rolled out in all participating BUs with real-time monitoring of results. This regional-based 


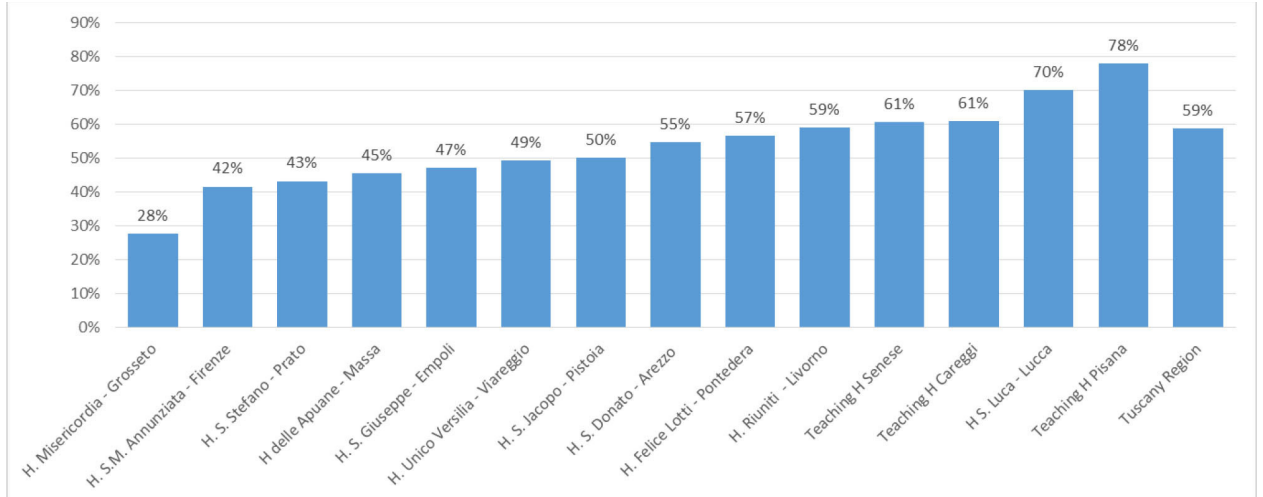

Figure 1 Percentage of iBR after mastectomy over total mastectomy for breast cancer across BUs in Tuscany Region (2019). *Source: Performance Evaluation System of Tuscany Region. ${ }^{54} \mathrm{H}$, Hospital.

study was designed as a continuous and routine data collection system, without a predefined ending date.

\section{Patient and public involvement}

Patients are involved in the study as active participants. The study is designed to collect the patients' voice, and surveys are addressed to them. During the pilot phase, patients contributed to improving the design and tools for data collection, providing suggestions via email during survey completion and feedback on comprehension of survey items. Currently, there is no formal patient representative group as part of the study group, or representatives from patient associations. Results of the study will be made available to all study participants via annual public reports published online.

\section{Participants}

Inclusion criteria are:

- Women over the age of 18 years.

- Malignant breast cancer (ICD-9-CM diagnosis code $174 *)$.
- Implant and autologous reconstruction.

- Immediate (one step and two step) breast reconstructive surgery at the site after a mastectomy.

- Unilateral or bilateral mastectomy including prophylactic mastectomy (ie, risk-reduction surgery) and mastectomy for aesthetic improvement.

- Primary breast cancer or breast tumour relapse including positive lymph nodes and disease residue (infiltration of the margins).

- Agreement to participate in the study (oral consent). Exclusion criteria are:

- Poor reconstructive candidate (as determined by breast surgeon and/or plastic surgeon).

- Tumours with characteristics not meeting the above inclusion criteria.

\section{Procedure}

Potential participants are presented the study information and may provide oral consent during one of their visits with breast surgeons or/and plastic surgeons preceding

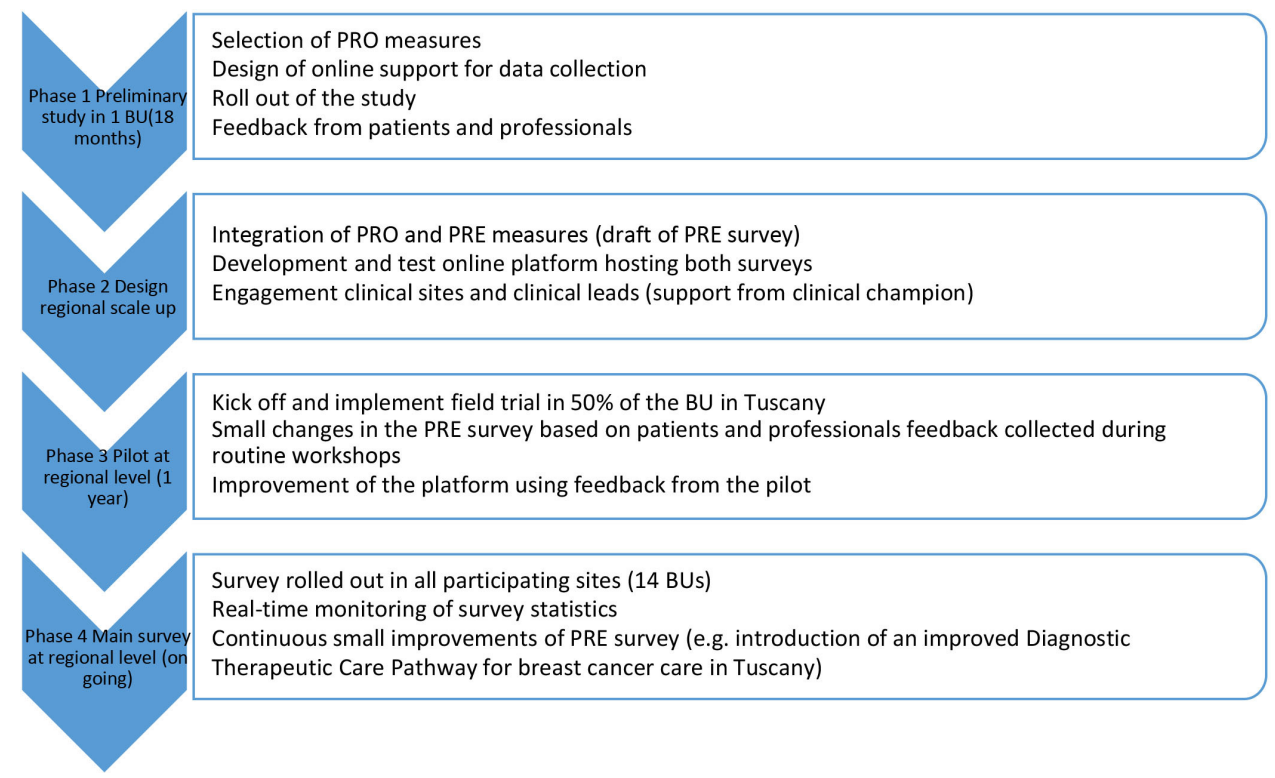

Figure 2 Workflow of study launch and roll-out. BU, Breast Unit; PRE, patient-reported experience; PRO, patient-reported outcome. 
the iBR (after the diagnostic phase is completed). If the patient provides consent to participate in the study, she will be enrolled by the physician.

Patients are identified and classified into two groups based on iBR timing:

- One-stage iBR group: insertion of permanent implant (prosthesis/mesh) or autologous tissue at initial surgery.

- Two-stage iBR group: insertion of a temporary tissue-expander, with a later planned definitive reconstruction.

- Patients can decide to leave the study anytime, either by replying to the survey invitation, sending an email to a dedicated email address or asking the clinician who enrolled her in the study to discontinue the invitations. All collected data will be analysed.

\section{Variables}

Primary outcome measures used are QoL (physical, psychological and sexual well-being in BR), and experience with care along the care pathways (from diagnosis to 12-month follow-up after reconstruction). Outcome variables are summarised in table 2.

\section{Patient-reported outcomes}

The BREAST-Q is a validated condition-specific PRO assessment tool, measuring various aspects of outcomes from the patient's perspective, developed at the Memorial Sloan Kettering Cancer Center and the University of British Columbia. ${ }^{356}$ Our study uses the pre/post-breast reconstructive modules of the BREAST-Q V.2.0 to assess patient satisfaction and health-related QoL across 10 domains. All scales of the BREAST-Q V.2.0 reconstructive module are administered. The validated Italian version of the BREAST-Q V.2.0 is used. The survey is web-based, administered after consultation with the surgical oncologist and/or plastic surgeon in charge of the iBR (baseline), and at 3 and 12 and months following completion of the reconstruction (follow-ups). The BREAST-Q is able to achieve high response rates, even with web-based administration. ${ }^{3788}$

\section{Self-rated general health}

Patients are asked about their perceived general health status in all questionnaires. The question is: 'In general, in the last week, would you say your health is....? Excellent/Very Good/Good/Fair/Bad'.

\section{Patient-reported experience}

PRE surveys were developed on the basis of previous work conducted at Management and Healthcare Laboratory on inpatient experience over the past decade. ${ }^{39-41}$ These PREMs cover experiences throughout the care pathway, targeted objectives and events of interest (eg, organisation and accessibility, information and communication, and coordination and continuity). The complete list of PRE questions are available in the online supplemental appenix 1. In particular:
- The first survey, administered prior to breast surgery, asks for information on the pathway from tumour diagnosis to treatment plan. There are questions regarding access and waiting times for examinations carried out, sources of information to understand the condition (ie, family physician, medical specialists, internet), the role of the general practitioners (GPs) in this phase of the pathway and any second opinions about the surgical indication.

- The first follow-up survey, administered 1 month after $\mathrm{BR}$, includes questions about experiences during the hospital stay. There are also questions addressing perceived waiting time before surgery, the role of the GP and rehabilitation-in particular regarding the early in-hospital rehabilitative practices.

- The second follow-up survey, administered 3 and 12 months after BR, includes questions focusing on the postoperative care pathway and the use of outpatient services and oncological support services (eg, psychoncologist, nutritionist, integrative medicine) and integration with primary care.

All surveys include additional free text questions, providing the opportunity to collect patients' views in their own words about issues which may not have been adequately covered in the closed response questions.

In addition to the outcome measures, the preoperative survey includes patient-reported biographical data, medical history and lifestyle habits. Further medical and surgical data are reported by clinicians during enrolment. This information is collected to characterise patients and to enable the comparability of PRO and PRE data through appropriate risk adjustment procedures. ${ }^{42}$ Table 3 provides details of the secondary variables collected from patients or clinicians at baseline.

\section{Sources of data and data management}

The entire data collection is managed digitally. Patient demographics, contact details and clinical data such as comorbidities, type of reconstruction and lymph node management are collected by healthcare staff (usually breast or plastic surgeons) during patient recruitment. Healthcare staff use a web-based platform to recruit and enrol eligible women after consent is obtained. These data build the enrolment dataset, which is password protected and visible only to staff responsible for enrolment. Each professional can only view patients they have enrolled.

A unique identifier is randomly generated by the web platform, enabling surveys to be sent anonymously. All surveys are sent directly to the patients via email or text message containing a link to the questionnaire. Patients can answer the surveys using either a smart phone, personal computer or tablet. Survey responses are stored in separate datasets and are linked to the enrolment dataset by the unique identifier. Biographical and basic lifestyle information are self-reported by patients in the baseline survey. Outcome and experience measures are reported by the patients following the timeline in table 2 . 


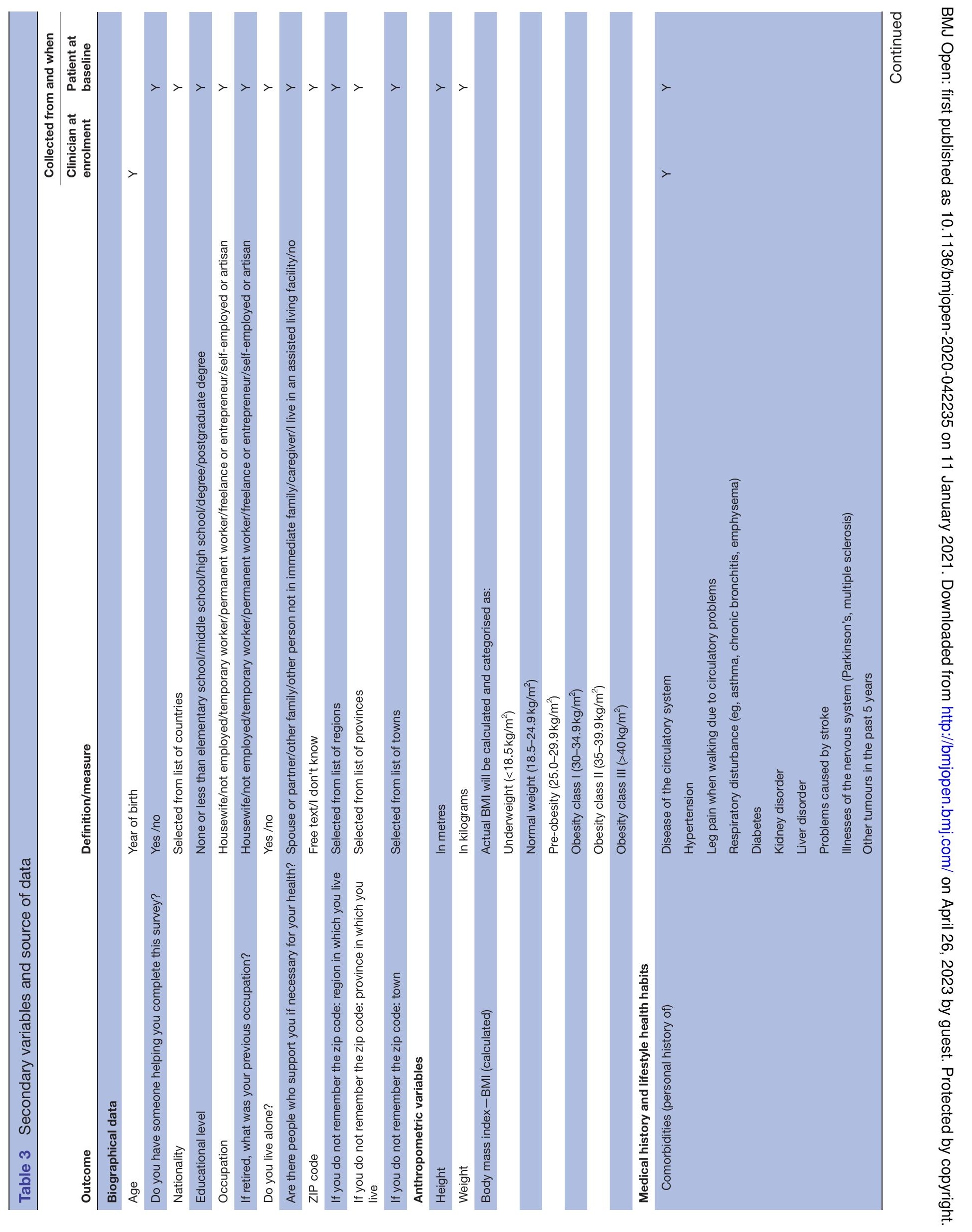


菏

产

离

등

ग

它

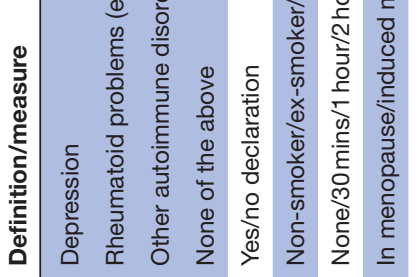

亏े

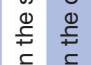

कृ

产

范

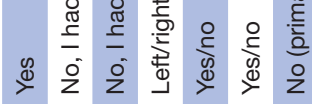

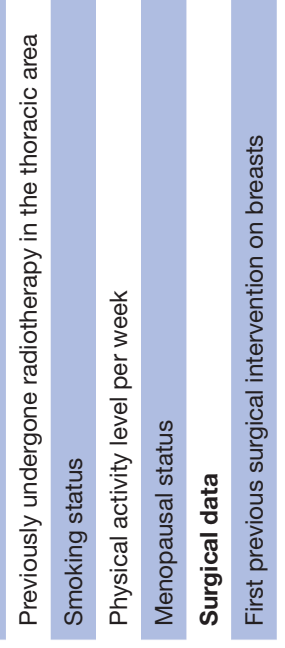

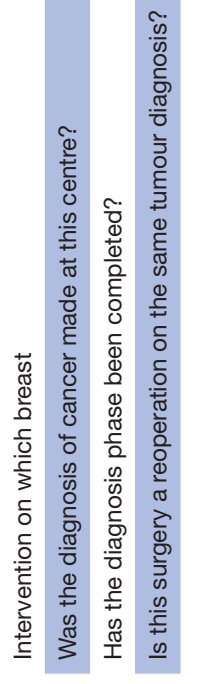

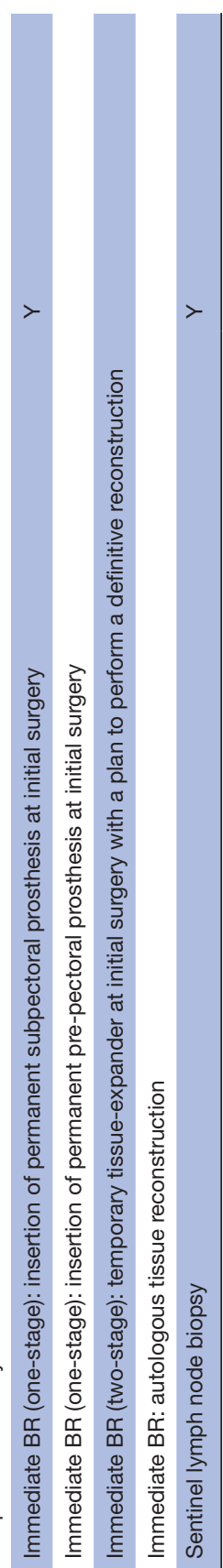

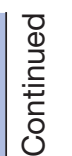

$\stackrel{\overrightarrow{\vec{O}}}{\vec{\theta}}$

$\frac{\bar{c}}{\overline{\frac{D}{5}}}$

일

$\vec{\circ}$

$\overrightarrow{\vec{\omega}}$

$\frac{\mathrm{O}}{\mathrm{O}}$

흉

กิ

’े

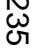

옥

$\exists$

@)

ฏ)

옴

웅

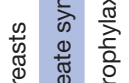

产

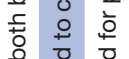

응

훙

菐

$\frac{\bar{y}}{3}$

응

$\stackrel{\circ}{3}$

ฏे

욱

을

N

N

W

잉

$\stackrel{\$}{\stackrel{9}{+口}}$

$\underset{7}{0}$

유

응

ত্

을. 
Names and contact data are deleted at completion of the last survey, when the last reminder is sent by email or text message, or when the patient drops out from the survey. All datasets reside on the servers of Scuola Superiore Sant'Anna, in Pisa (Italy). The data collection and storage systems (ie, web platform, servers) are set according to the European General Data Protection Regulation 679 (2016).

This mode of administration allows automatic email and text message reminders to non-responders, and invitations for follow-up questionnaires to study participants.

The electronic data entry enables automated transformation and analysis, allowing real-time reporting of aggregate PREMs and PROMs for each provider. Tables and bar graphs are generated so that clinicians enrolling patients can visualise (for each time point) aggregate data in a simple format (figure 3). The real-time data presentation, can be used as a management tool to monitor and develop service quality, including the impact of any organisational changes. Such data can also be presented to staff as part of quality improvement discussions, performance reviews and in highlighting achievements. The aggregate data can be used by clinicians to inform decision-making for women considering iBR.

Further data management and data analysis are performed using SAS V.9.4 and Stata V.15.

\section{Study size}

The study aims to systematically include patients meeting the eligibility criteria undergoing iBR surgery in the BUs of Tuscany. Findings from participation in longitudinal studies $^{43}{ }^{44}$ suggest an expected first year enrolment rate of $30 \%$ of patients. In 2019 , the first year of operation, about 220 women were enrolled from around 700 eligible patients-a $31 \%$ enrolment rate. An increase in participation is expected over time as familiarity with the digital survey administration model grows. ${ }^{45}$ Previous studies indicated a drop-out rate at follow-up ranging between $30 \%$ and $70 \% .{ }^{46}$ Thus, around $50 \%$ follow-up drop-out rate is also expected.

\section{Potential bias}

During the longitudinal study, sample attrition rates are calculated for each follow-up period. Based on the pilot study, an attrition rate of $35 \%$ is estimated at 12 months. We will investigate if attrition over time is random or systematic. Previous research showed that patient characteristics can affect willingness and ability to participate in longitudinal studies. ${ }^{46} 47$

Selective non-responses are investigated in the first years of study roll-out to explore self-selection in study participation. We will verify ex-post that respondents are not significantly different from the total relevant population, based on common information available in the sample list (for recruited women) and from patient-level hospital discharge records (for the population). Type of reconstruction and type of mastectomy will be considered for additional sensitivity analyses. 


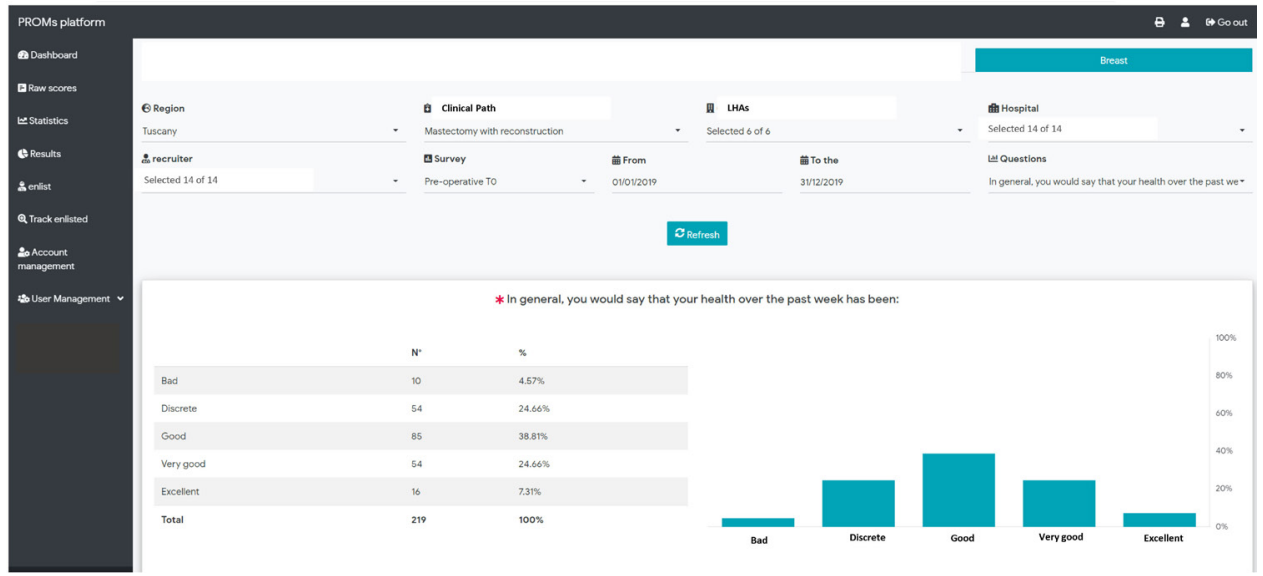

Figure 3 Real-time data visualisation. LHAs, local health authorities; PROMs, patient-reported outcome measures.

\section{Statistical analyses}

All measures are summarised using descriptive analysis, overall and split by type of reconstruction. To compare possible differences between groups at baseline, one-way analysis of variance (ANOVA), Tukey's post hoc analysis and the Kruskal-Wallis test is used according to data distribution.

To answer the first aim of the study-to describe PROs for the study population, including the relationship to surgical outcomes_ANOVA for repeated measures is employed to test for differences over time. PRO scores are calculated according to the BREAST-Q scoring manual, which also defines methods for handling missing items. Crude PRO scores are analysed using paired t-test and Wilcoxon matched-pairs signed rank-tests, for parametric and non-parametric data, respectively. We conduct a preoperative and postoperative comparison for each patient group to evaluate score changes over time, and a group score comparison to determine whether differences in BREAST-Q scores are significant between types of reconstruction. We also assess specific patterns in PROs considering significant population subgroups (eg, by age, comorbidities, body mass index level and smoking habits).

Additionally, econometric models will be used to analyse patient and hospital characteristics associated with PRO dimensions (satisfaction with surgery, psychological impact, physical and sexual well-being) and PRE results. Scores will be risk-adjusted by those factors significantly related to the outcome measure with heterogeneous distribution across BUs. As the data are collected anonymously, statistical analyses will not consider any clinical, biological and therapeutic characteristics of patients not collected during the study.

To answer the second aim of the study-to analyse PRE along the pathway-we evaluate differences in patient experiences along the care pathway, including dimensions of integrated care: organisation and accessibility, information and communication, and coordination and continuity.
Finally—to monitor providers' participation in patientreported data collection-enrolment and response rate at baseline are measured across providers to support higher participation rates over time.

Data analysis is performed by the Management and Healthcare Laboratory of the Scuola Superiore Sant'Anna Pisa (Italy). Health professionals are involved in the analysis and the discussion of data both individually and collectively during specific workshops organised throughout the year.

\section{DISCUSSION (KEY RESULTS, LIMITATIONS, INTERPRETATIONS, GENERALISABILITY)}

This study provides the first regional-level routinely collected patient-reported information on breast cancer care, integrating outcome and experience data in Italy. It addresses important gaps in the development and use of PROs and PREs through routinely monitoring outcomes and experiences that matter to patients with breast cancer.

The resulting data can be used by health systems' stakeholders to evaluate and improve care and to inform discussions with patients about treatment options, as well as by scholars to study the relationships between structure, process, outcomes and experiences in breast cancer care.

While PROs and PREs are increasingly used in quality measurement and improvement, most available studies are on a small scale and over limited time. Studies also typically focus on a single incident of care, potentially with pre-surgical and post-surgical measures, with assessments made from the perspective of healthcare organisations or clinicians.

This study addresses these current conceptual weaknesses: data collection is systematic, at scale and continuous; the multicentre and large scale study allows benchmarking across providers; monitoring both PROMs and PREMs for at least 12 months provides richer insights into outcomes over time and experiences in access and use of services along the care pathway; and, following the multiprovider and cross-setting patient 
pathway overcomes the 'silo-vision' related to a specific care setting or organisation. ${ }^{48}{ }^{49}$ The resulting data can be triangulated with existing health system intelligence to help investigate the most important factors determining patient experience, health status and QoL over time.

The completely digital administration and management of the survey provides some notable features. Primarily, the use of electronic collection and web-reporting enables the larger scale collection and analysis of patientreported data, with high acceptability and convenience for respondents. The digital administration of questionnaires and reporting of data is low cost and easy to use, and can offer multiple additional functions, particularly when compared with postal or telephone surveys. This digital model also enables real-time reporting of data (after a minimum number of questionnaires have been collected), providing value back to providers. This can incentivise provider participation, as well as enabling patient benefit where data returns are used effectively as a management tool. ${ }^{34}$

Additionally, the personalised web-based surveys enable patients to reply autonomously, without support from healthcare workers (who may influence response tendencies). The fully digital process may provide a barrier to participation among some-particularly older-women, although national data show that people aged 64 years and older are increasingly familiar with digital technologies. ${ }^{50}$ Sensitivity analyses applied to data gathered during the pilot do not suggest respondents are selected in any way based on age, comorbidities or nationality.

Other challenges relate to the scale and scope of provider involvement. First, although multicentre, the study is implemented in only one Italian region, which may limit generalisability of results. Second, the data collection model depends on clinicians and other professionals adequately informing and enrolling patients. Their continuing enthusiasm cannot be guaranteed. ${ }^{51}$ Managerial levers are needed to enhance their participation: for instance, yearly indicators on the enrolment rate and on the preoperative response rate, with specific targets for the healthcare managers, have been introduced to this end. Routine monitoring is performed to identify changes in enrolment over time, enabling targeted communication actions towards professionals where required. Another strategy we are using to enhance professional enrolment is regular disclosure of enrolment data and response rates at regional breast cancer network meetings, through which benchmarking and reputational levers can encourage enrolments. As already noted, patient drop-outs can limit the power of the analysis, even where strategies to improve response rates are in place, such as individual patient reminders.

Finally, without a fully randomised recruitment process, there is a risk of bias in patient enrolment.

\section{ETHICS AND DISSEMINATION}

Ethics and privacy issues were addressed with the Tuscan regional office that provides support for health research and clinical trials, supervises the protection of personal data in the health sector and supports the ethics committees. The formal documents were prepared and submitted to the four Tuscan ethics committees. Documents include the Participant Information Sheet, copy of the questionnaires, list of additional information collected by the clinical professionals during patient enrolment, study protocol and data management plan. The study obtained the nihil obstat from the Ethics Committees of: (1) Vasta Area Sud-Est on 20 November 2017, Area Vasta Centro on 21 November 2017 and Area Vasta Nord-Ovest on 07 December 2017. The Paediatric Ethics Committees provided a certification of notice. In compliance with the national guidelines of the Italian Data Protection Authority ${ }^{52}$ and according to the regional laws that regulate patient surveys (online supplemental sheet 12 appendix $\mathrm{A}$, and $\mathrm{B}),{ }^{53}$ PROMs were considered regulated as other patient surveys, such as PREMs. Financial support for the conceptualisation, design and implementation of the study was granted by Direzione Diritti di cittadinanza e coesione sociale of Tuscany Region. The funder is informed but does not have ultimate authority over the collection, management, analysis, interpretation of data and writing of reports.

The protocol will be disseminated via the study web page available at https:// www. santannapisa.it/it/ ricerca/progetti/ indagini-proms-nella-chirurgiaper-tumore-al-seno.

Dissemination of results will be via periodic report to Tuscany Region and professionals involved using aggregate data. In addition, the results of the study will be published in peer-reviewed journals and presented to appropriate conferences.

\section{Author affiliations}

${ }^{1}$ Department EMbeDS, Institute of Management, MeS Management and Health Laboratory, Sant'Anna School of Advanced Studies, Pisa, Toscana, Italy

${ }^{2}$ Breast Cancer Centre, Azienda Ospedaliero Universitaria Pisana, Pisa, Toscana, Italy

\section{Twitter Sabina De Rosis @SabinaDeRosis}

Contributors SN supervised the initiative and its implementation, and critically reviewed the manuscript. SDR supervised the initiative and its implementation and contributed to writing the manuscript. AMM coordinated the initiative and contributed to defining its organisation and structure from the pilot phase, and critically reviewed the manuscript. FF coordinated the initiative and contributed to defining its organisation and structure from the pilot phase, and drafted the manuscript. KJG contributed to writing and editing the manuscript. MR collaborated in the study design, acted as the clinical champion of the initiative among the other BUs and critically reviewed the manuscript. MG and DM supported the team from the pilot phase, provided specific insights about the clinical and organisational aspects of breast cancer care, and critically reviewed the manuscript.

Funding The work leading to the development of the protocol has received funding from Regione Toscana under grant agreement NET-2016-02363853-4 (Project CARE-NETS) Bando della Ricerca finalizzata 2016, Italian Ministry of Health. Financial support for the conceptualisation, design and implementation of the study was granted by Direzione Diritti di cittadinanza e coesione sociale of Tuscany Region. 
Disclaimer The funder is informed but does not have ultimate authority over the collection, management, analysis, interpretation of data and writing of reports.

Competing interests None declared.

Patient consent for publication Not required

Provenance and peer review Not commissioned; externally peer reviewed.

Supplemental material This content has been supplied by the author(s). It has not been vetted by BMJ Publishing Group Limited (BMJ) and may not have been peer-reviewed. Any opinions or recommendations discussed are solely those of the author(s) and are not endorsed by BMJ. BMJ disclaims all liability and responsibility arising from any reliance placed on the content. Where the content includes any translated material, BMJ does not warrant the accuracy and reliability of the translations (including but not limited to local regulations, clinical guidelines, terminology, drug names and drug dosages), and is not responsible for any error and/or omissions arising from translation and adaptation or otherwise.

Open access This is an open access article distributed in accordance with the Creative Commons Attribution Non Commercial (CC BY-NC 4.0) license, which permits others to distribute, remix, adapt, build upon this work non-commercially, and license their derivative works on different terms, provided the original work is properly cited, appropriate credit is given, any changes made indicated, and the use is non-commercial. See: http://creativecommons.org/licenses/by-nc/4.0/.

\section{ORCID iDs}

Francesca Ferrè http://orcid.org/0000-0001-5781-517X

Sabina De Rosis http://orcid.org/0000-0002-8781-401X

\section{REFERENCES}

1 AIOM - AIRTUM - FONDAZIONE AIOM. I numeri del cancro in Italia 2019, 2019: 1-388.

2 Howard MA, Sisco M, Yao K, et al. Patient satisfaction with nipplesparing mastectomy: a prospective study of patient reported outcomes using the BREAST-Q. J Surg Oncol 2016;114:416-22.

3 Cardoso F, Kyriakides S, Ohno S. Early breast cancer: ESMO clinical practice guidelines for diagnosis treatment and follow-up, 2019.

4 Ghilli M, Mariniello MD, Camilleri V, et al. Proms in post-mastectomy care: patient self-reports (BREAST-Q) as a powerful instrument to personalize medical services. Eur J Surg Oncol 2020;46:1034-40.

5 Devlin NJ, Appleby J, Buxton M. Getting the most out of PROMs: health outcomes and NHS decision-making, Nancy Devlin and John Appleby. The King's Fund, 2010.

6 Slawomirski L, van den Berg M, Karmakar-Hore S. Patient-reported indicator survey (Paris): aligning practice and policy for better health outcomes. World Med J 2018;64.

7 Coulter A. Can patients assess the quality of health care? BMJ 2006;333:1-2.

8 Klazinga NS, Fujisawa R. Measuring patient experiences (PREMS) progress made by the OECD and its member countries between 2006 and 2016, 2017.

9 Coulter A, Fitzpatrick R, Cornwell J. The point of care. Measures of patients' experience in hospital: purpose, methods and uses, 2009

10 Doyle C, Lennox L, Bell D. A systematic review of evidence on the links between patient experience and clinical safety and effectiveness. BMJ Open 2013;3:e001570.

11 Wang DE, Tsugawa Y, Figueroa JF, et al. Association between the centers for Medicare and Medicaid services Hospital StAR rating and patient outcomes. JAMA Intern Med 2016;176:848-50.

12 Chen CM, Cano SJ, Klassen AF, et al. Measuring quality of life in oncologic breast surgery: a systematic review of patient-reported outcome measures. Breast J 2010;16:587-97.

13 Snyder CF, Aaronson NK. Use of patient-reported outcomes in clinical practice. Lancet 2009;374:369-70.

14 Black N, Burke L, Forrest CB, et al. Patient-reported outcomes: pathways to better health, better services, and better societies. Qual Life Res 2016;25:1103-12.

15 Basch E, Deal AM, Dueck AC, et al. Overall survival results of a trial assessing patient-reported outcomes for symptom monitoring during routine cancer treatment. JAMA 2017;318:197-8.

16 Black N. Patient reported outcome measures could help transform healthcare. BMJ 2013;346:f167.

17 Coronini-Cronberg S, Appleby J, Thompson J. Application of patient-reported outcome measures (PROMs) data to estimate cost-effectiveness of hernia surgery in England. $J R$ Soc Med 2013;106:278-87.
18 van Egdom LSE, Oemrawsingh A, Verweij LM, et al. Implementing patient-reported outcome measures in clinical breast cancer care: a systematic review. Value Health 2019;22:1197-226.

19 Hibbard JH, Mahoney E, Sonet E. Does patient activation level affect the cancer patient journey? Patient Educ Couns 2017;100:1276-9.

20 Atisha DM, Rushing CN, Samsa GP, et al. A national snapshot of satisfaction with breast cancer procedures. Ann Surg Oncol 2015;22:361-9.

21 Jeevan R, Cromwell DA, Browne JP, et al. Findings of a national comparative audit of mastectomy and breast reconstruction surgery in England. J Plast Reconstr Aesthet Surg 2014;67:1333-44.

22 OECD. Measuring what matters: the patient-reported indicator surveys, 2019.

23 Sitzia J, Wood N. Development and evaluation of a questionnaire to assess patient satisfaction with chemotherapy nursing care. Europ $J$ Oncol Nurs 1999;3:126-40.

24 Ruban PU, Wulff CN, Sperling CD, et al. Patient evaluation of breast cancer follow-up: a Danish survey. Patient Educ Couns 2018;101:99-104.

25 Grunfeld E, Fitzpatrick R, Mant D, et al. Comparison of breast cancer patient satisfaction with follow-up in primary care versus specialist care: results from a randomized controlled trial. $\mathrm{Br} J$ Gen Pract 1999;49:705.

26 Kimman ML, Bloebaum MM, Dirksen CD, et al. Patient satisfaction with nurse-led telephone follow-up after curative treatment for breast cancer. BMC Cancer 2010;10:174.

27 McWilliam CL, Brown JB, Stewart M. Breast cancer patients' experiences of patient-doctor communication: a working relationship. Patient Educ Couns 2000;39:191-204.

28 Hermens R, van Overveld LFJ, Takes RP, et al. PROMs and PREMs in Dutch integrated head and neck cancer care. JCO 2017;35:6570.

29 The University of Edinburgh. Edinburgh cancer informatics, 2020 Available: https://blogs.ed.ac.uk/cancer-data/category/proms/

30 Withers K, Puntoni S, Palmer R, et al. OP96 standardizing collection of patient-reported experience measures to drive service improvement in Wales. Int J Technol Assess Health Care 2018;34:35.

31 Holch P, Absolom K, Brooke C. Advances in patient reported outcomes: integration and innovation. J Patient-Reported Outcomes 2020;4:28.

32 Wilson ARM, Marotti L, Bianchi S, et al. The requirements of a specialist breast centre. Eur J Cancer 2013;49:3579-87.

33 Gilmore KJ, Pennucci F, De Rosis S, et al. Value in healthcare and the role of the patient voice. Healthc Pap 2019;18:28-35.

34 De Rosis S, Pennucci F, Nuti S. From experience and outcome measurement to the health professionals' engagement. Micro Macro Mark 2019;3:493-520.

35 Pusic AL, Klassen AF, Scott AM, et al. Development of a new patientreported outcome measure for breast surgery: the BREAST-Q. Plast Reconstr Surg 2009;124:345-53.

36 Cano SJ, Klassen AF, Scott AM, et al. The BREAST-Q: further validation in independent clinical samples. Plast Reconstr Surg 2012;129:293-302

37 Fuzesi S, Cano SJ, Klassen AF, et al. Validation of the electronic version of the BREAST-Q in the army of women study. Breast 2017;33:44-9.

38 Cohen WA, Mundy LR, Ballard TNS, et al. The BREAST-Q in surgical research: a review of the literature 2009-2015. J Plast Reconstr Aesthet Surg 2016;69:149-62.

39 Murante AM, Vainieri M, Rojas D, et al. Does feedback influence patient - professional communication? Empirical evidence from Italy. Health Policy 2014;116:273-80.

40 Murante AM, Seghieri C, Brown A, et al. How do hospitalization experience and institutional characteristics influence inpatient satisfaction? A multilevel approach. Int J Health Plann Manage 2014;29:247-60.

41 Nuti S, Murante AM. L'esperienza e la soddisfazione dei pazienti oncologici per i servizi sanitari ricevuti in Toscana. In: Giunti OS, ed. Organizzazioni Speciali, editor. La valutazione di qualit nella rete oncologica Toscana Dalle Raccomandazioni cliniche ITT agli indicatori del percorso assistenziale. Firenze, Italy, 2009: 114-25.

42 Nuttall D, Parkin D, Devlin N. Inter-provider comparison of patientreported outcomes: developing an adjustment to account for differences in patient case mix. Health Econ 2015;24:41-54

43 Morton SMB, Bandara DK, Robinson EM, et al. In the 21st century, what is an acceptable response rate? Aust $N Z \mathrm{~J}$ Public Health 2012;36:106-8.

44 Galea S, Tracy M. Participation rates in epidemiologic studies. Ann Epidemiol 2007;17:643-53.

45 Van Gelder M, Bretveld RW, Roeleveld N. Practice of epidemiology web-based questionnaires the future in epidemiology? $\mathrm{Am} \mathrm{J}$ Epidemiol 2010;172. 
46 Gustavson K, von Soest T, Karevold E, et al. Attrition and generalizability in longitudinal studies: findings from a 15-year population-based study and a Monte Carlo simulation study. BMC Public Health 2012;12:918.

47 Gaertner B, Seitz I, Fuchs J, et al. Baseline participation in a health examination survey of the population 65 years and older: who is missed and why? BMC Geriatr 2016;16:21.

48 et alNuti S, Noto G, Vola F. Let's play the patients music. Manag Decis [online], 2018. Available: https://www.emeraldinsight.com/doi/ 10.1108/MD-09-2017-0907

49 Nuti S, De Rosis S, Bonciani M, et al. Rethinking healthcare performance evaluation systems towards the People-Centredness approach: their pathways, their experience, their evaluation. Healthc Pap 2017;17:56-64.

50 Total Digital Audience February 2020 - Audiweb http://www.audiweb. it/news/comunicati-stampa/total-digital-audience-Febbraio2020.html

51 Lungu DA, Pennucci F, De Rosis S, et al. Implementing successful systematic patient reported outcome and experience measures
(PROMs and PREMs) in robotic oncological surgery-The role of physicians. Int J Health Plann Manage 2020;35:773-87.

52 Garante per la Protezione dei Dati Personali. Guidelines on processing personal data to perform customer satisfaction surveys in the health care sector [online]. Italy's Official J 2011 https://www. garanteprivacy.it/home/docweb/-/docweb-display/docweb/3853781

53 Regione Toscana. Trattamento dei dati sensibili e giudiziari da parte della Regione Toscana, aziende sanitarie, enti, aziende e agenzie regionali e soggetti pubblici nei confronti dei quali la Regione esercita poteri di indirizzo e controllo, 6/R [online]. Regione Toscana. Available: http://raccoltanormativa.consiglio.regione. toscana.it/articolo?urndoc=urn:nir:regione.toscana:legge:2006-0403;13

54 Performance evaluation system of Tuscany Region. Available: https:// performance.santannapisa.it/pes/start/start.php [Accessed 20 May 2020].

55 Senonetwork. Available: http://www.senonetwork.it/centri [Accessed 17 Apr 2020]. 